\title{
van der Waals like behaviour of topological AdS black holes in massive gravity
}

\author{
S. H. Hendi, ${ }^{1,2}$ R. B. Mann, ${ }^{3}$ S. Panahiyan, ${ }^{1,4}$ and B. Eslam Panah ${ }^{1}$ \\ ${ }^{1}$ Physics Department and Biruni Observatory, College of Sciences, Shiraz University, Shiraz 71454, Iran \\ ${ }^{2}$ Research Institute for Astronomy and Astrophysics of Maragha (RIAAM), Maragha, Iran \\ ${ }^{3}$ Department of Physics and Astronomy, University of Waterloo, \\ 200 University Avenue West, Waterloo, Ontario, Canada N2L 3G1 \\ ${ }^{4}$ Physics Department, Shahid Beheshti University, Tehran 19839, Iran
}

\begin{abstract}
Motivated by recent developments in black hole thermodynamics, we investigate van der Waals phase transitions of charged black holes in massive gravity. We find that massive gravity theories can exhibit strikingly different thermodynamic behaviour compared to that of Einstein gravity, and that the mass of the graviton can generate a range of new phase transitions for topological black holes that are otherwise forbidden.
\end{abstract}

PACS numbers: 04.70.Dy, 04.40.Nr, 04.20.Jb, 04.70.Bw

Introduction- Understanding the quantum behaviour of gravity could be related to the possible mass of the graviton. The consistency of including this in the context of extending general relativity has been a long-standing basic physical question of classical field theory. Although the primitive linear theory of massive gravity [1] contains Boulware-Deser ghost modes [2], a nonlinear generalization that is ghost free to all orders has recently been constructed [3] by de Rham, Gabadadze and Tolley (dRGT massive theory). dRGT is stable, enjoys the absence of Boulware-Deser ghost [4], and has yielded a number of intriguing results in terms of its cosmological behaviour and black hole solutions [5]. The mass terms are produced by consideration of a reference metric, which plays a crucial role in the construction of dRGT [6]. Motivated by applications of gauge/gravity duality, Vegh proposed a new reference metric in which the graviton behaves like a lattice excitation and exhibits a Drude peak [7]. This theory is also ghost-free and stable [8], and $d$-dimensional $(d \geq 3)$ black hole solutions in the presence of linear and nonlinear electrodynamics with van der Waals like behavior have been obtained [9]. Higher curvature generalizations have also been constructed [10]. Although some classes of nonlinear massive gravity theories are Lorentz-violating and bear a close relation to Horava-Lifshitz gravity [11], it was shown that there are Lorentz invariant versions of nonlinear massive gravity as well [3]. Massive gravity is also motivated by observation. Obtaining an empirical upper limit on the mass of the graviton remains an outstanding challenge (for more details see [12]), one that should soon be attainable once recent LIGO results [13] are improved and expanded. In this regard, one may use the results of Refs. [14] and [15] to obtain a bound on the energy flux emitted from a binary pulsar and on the propagation speed of the graviton.

Here, we consider a class of dRGT theories, which we regard as the minimal modification of general relativity that yields a massive graviton [3]. We demonstrate that black holes of non-spherical topology can exhibit van der Waals phase transitions in dRGT like gravity. Such tran- sitions are forbidden in standard Einstein gravity and also higher curvature theories such as Lovelock gravity.

The study of black hole thermodynamics began with the pioneering work of the Hawking and Page [16] that indicated anti-de Sitter (AdS) black holes can undergo phase transitions. Asymptotically AdS black holes have been of special interest since they admit a gauge/gravity duality description, and their thermodynamics plays a crucial role in constructing a consistent theory of quantum gravity [17]. Indeed, this duality can be applied to a qualitative study of the behaviour of various condensed matter phenomena [18]. Substantial progress was recently made when van der Waals behaviour of asymptotically AdS charged black holes was observed [19]. Based on the canonical ensemble, a small-large AdS black hole phase transition analogous to the liquid-gas phase transition in a thermodynamic system was discovered. A number of significant results were subsequently obtained, including the existence of triple points 20], reentrant phase transitions [21], and analogous Carnot-cycle heat engines [22]. These properties established a connection between black hole thermodynamics and everyday "chemical" thermodynamics, known as "black hole chemistry". It likewise trigged investigations into the implications for gauge/gravity duality, such as holographic superconductors [23], the Kerr/CFT correspondence 24], and holographic entanglement entropy [25] (see 26] for a review).

However, van der Waals behaviour (and its applications) in Einstein gravity are seen only for AdS black holes with spherical horizon topology [19]; no such behaviour takes place for AdS black holes with flat or hyperbolic horizons (no real critical point). Such reports motivate one to look for an extension of Einstein gravity to a modified version, in which van der Waals behaviour is seen not only for spherical AdS black holes, but for AdS black holes with different horizon curvature. In what follows, we demonstrate that black holes in massive gravity exhibit van der Waals behaviour independent of the choice of horizon curvature. This relaxes the constraint on the topological structure of the solutions, allowing the possibility of conducting studies in the context of black 
hole chemistry, the AdS/CFT correspondence, and the non-relativistic AdS/CMT correspondence, regardless of horizon geometry.

Basic Setup- The $d$-dimensional action of Einstein- $\Lambda$ massive gravity with a $U(1)$ gauge field is

$\mathcal{I}=\frac{-1}{16 \pi} \int d^{d} x \sqrt{-g}\left(\mathcal{R}-2 \Lambda-\mathcal{F}+m^{2} \sum_{i}^{4} c_{i} \mathcal{U}_{i}(g, f)\right)$,

in which $\mathcal{R}$ is the scalar curvature of the metric $g_{\mu \nu}, \Lambda$ is the negative cosmological constant and $\mathcal{F}=F_{\mu \nu} F^{\mu \nu}$ is the Maxwell invariant, where $F_{\mu \nu}=\partial_{\mu} A_{\nu}-\partial_{\nu} A_{\mu}$ is the electromagnetic tensor with gauge potential $A_{\mu}, m$ is massive parameter, and $f_{\mu \nu}$ is a fixed symmetric tensor. The $c_{i}$ 's are constants and the $\mathcal{U}_{i}$ 's are symmetric polynomials of the eigenvalues of the $d \times d$ matrix $\mathcal{K}_{\nu}^{\mu}=\sqrt{g^{\mu \alpha} f_{\alpha \nu}}$, where

$$
\begin{aligned}
& \mathcal{U}_{1}=[\mathcal{K}], \quad \mathcal{U}_{2}=[\mathcal{K}]^{2}-\left[\mathcal{K}^{2}\right], \\
& \mathcal{U}_{3}=[\mathcal{K}]^{3}-3[\mathcal{K}]\left[\mathcal{K}^{2}\right]+2\left[\mathcal{K}^{3}\right], \\
& \mathcal{U}_{4}=[\mathcal{K}]^{4}-6\left[\mathcal{K}^{2}\right][\mathcal{K}]^{2}+8\left[\mathcal{K}^{3}\right][\mathcal{K}]+3\left[\mathcal{K}^{2}\right]^{2}-6\left[\mathcal{K}^{4}\right] .
\end{aligned}
$$

Variation of the action (11) with respect to the metric tensor, $g_{\mu \nu}$, and the Faraday tensor, $F_{\mu \nu}$, leads to

$$
\begin{aligned}
G_{\mu \nu}+\Lambda g_{\mu \nu}+m^{2} \chi_{\mu \nu} & =-2\left(F_{\mu \rho} F_{\nu}^{\rho}-\frac{1}{4} g_{\mu \nu} \mathcal{F}\right) \\
\partial_{\mu}\left(\sqrt{-g} F^{\mu \nu}\right) & =0
\end{aligned}
$$

in which $G_{\mu \nu}$ is the Einstein tensor and $\chi_{\mu \nu}$ is

$$
\begin{aligned}
& \chi_{\mu \nu}=\frac{c_{1}}{2}\left(\mathcal{K}_{\mu \nu}-\mathcal{U}_{1} g_{\mu \nu}\right)-\frac{c_{2}}{2}\left(\mathcal{U}_{2} g_{\mu \nu}-2 \mathcal{U}_{1} \mathcal{K}_{\mu \nu}+2 \mathcal{K}_{\mu \nu}^{2}\right) \\
& -\frac{c_{3}}{2}\left(\mathcal{U}_{3} g_{\mu \nu}-3 \mathcal{U}_{2} \mathcal{K}_{\mu \nu}+6 \mathcal{U}_{1} \mathcal{K}_{\mu \nu}^{2}-6 \mathcal{K}_{\mu \nu}^{3}\right)-\frac{c_{4}}{2} \times \\
& \left(\mathcal{U}_{4} g_{\mu \nu}-4 \mathcal{U}_{3} \mathcal{K}_{\mu \nu}+12 \mathcal{U}_{2} \mathcal{K}_{\mu \nu}^{2}-24 \mathcal{U}_{1} \mathcal{K}_{\mu \nu}^{3}+24 \mathcal{K}_{\mu \nu}^{4}\right) .
\end{aligned}
$$

In order to obtain AdS topological static charged black holes in massive gravity, we consider the metric of $d=$ $(n+2)$-dimensional spacetime in the following form

$$
d s^{2}=-\psi(r) d t^{2}+\frac{d r^{2}}{\psi(r)}+r^{2} h_{i j} d x_{i} d x_{j},
$$

where $i, j=1,2,3, \ldots, n$ and $h_{i j} d x_{i} d x_{j}$ is a spatial metric of constant curvature $d_{2} d_{3} k$ and volume $\mathcal{V}_{d_{2}}$, where $d_{i}=d-i$. The reference metric $f_{\mu \nu}$ is related to the spatial components $h_{i j}$ of the spacetime line element. Accordingly, we employ the ansatz $f_{\mu \nu}=\operatorname{diag}\left(0,0, c^{2} h_{i j}\right)$ yielding

$$
\mathcal{U}_{j}=\frac{c^{j}}{r^{j}} \Pi_{k=2}^{j+1} d_{k},
$$

where $c$ is a positive constant [27]. This choice of reference metric preserves general covariance in the temporal and radial coordinates, but not in the transverse spatial coordinates [7] and so graviton mass terms will have a Lorentz-breaking property.
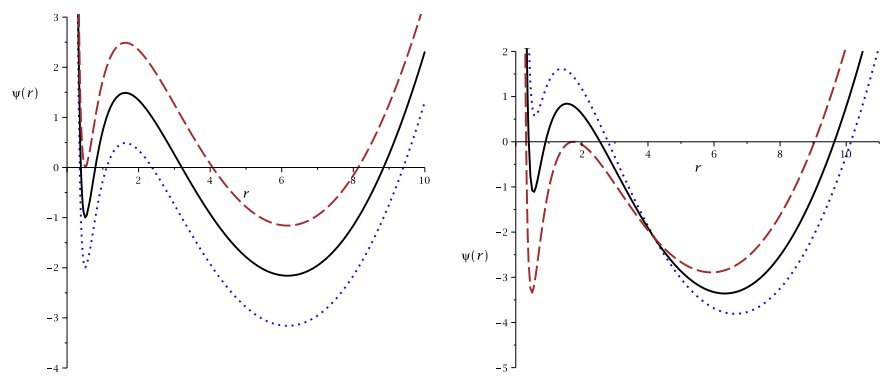

Figure 1: $\psi(r)$ versus $r$ for $\Lambda=-1, d=4, q=1.7, c=1.00$, $c_{1}=-2.00, c_{2}=3.18, c_{3}=4.00$ and $m_{0}=30$. Left panel: $m=2.1, k=-1$ (dotted line), $k=0$ (continuous line) and $k=1$ (dashed line). Right panel: $k=-1, m=2.16$ (dotted line), $m=2.12$ (continuous line) and $m=2.07$ (dashed line).

Setting $d=4$, the gauge potential ansatz $A_{\mu}=h(r) \delta_{\mu}^{0}$ yields from (3) $F_{t r}=\frac{q}{r^{2}}$ as the only nonzero component of electromagnetic field tensor, in which $q$ is an integration constant and is related to the electrical charge.

The field equations then yield

$$
\psi(r)=k-\frac{m_{0}}{r}-\frac{\Lambda r^{2}}{3}+\frac{q^{2}}{r^{2}}+m^{2} \mathcal{A},
$$

where $\mathcal{A}=\frac{c c_{1}}{2} r+c^{2} c_{2}+\frac{c^{3} c_{3}}{r}$. The quantity $m_{0}$ is an integration constant that is related to the total mass of this black hole. We note that for zero graviton mass $(m=$ 0 ), the solution (7) reduces to the Reissner-Nordström black hole in 4-dimensions. Calculations of the Ricci and Kretschmann scalars indicate a diverge at the origin $\left(\lim _{r \rightarrow 0} R=\infty\right.$ and $\left.\lim _{r \rightarrow 0} R_{\alpha \beta \gamma \delta} R^{\alpha \beta \gamma \delta} \longrightarrow \infty\right)$; as $r \longrightarrow \infty$ we find $R_{\alpha \beta \gamma \delta} \rightarrow \frac{\Lambda}{3}\left(g_{\alpha \gamma} g_{\beta \delta}-g_{\beta \gamma} g_{\alpha \delta}\right)$, confirming that the solution is asymptotically AdS.

To study the effects of the massive terms on our solutions, we can investigate the roots of the metric function $(\psi(r)=0)$. In massive gravity, it is possible for there to be as many as four real roots in all horizon topologies: spherical $(k=1)$, flat $(k=0)$ and hyperbolic $(k=-1)$, and we illustrate sample results in table I (see also Fig. 11). The existence of more than two roots for the metric function is due to the presence of the massive terms. Such multi-horizon solutions have been of interest in understanding anti-evaporation processes [28]. We postpone a discussion of the causal and geodesic structures of this class of solutions for future work, concentrating on their thermodynamic behaviour.

Thermodynamics in the extended phase space- In extended phase space, the cosmological constant is regarded as a thermodynamic variable corresponding to pressure, with $P=-\frac{\Lambda}{8 \pi}$. This postulate leads to an interpretation of the black hole mass as enthalpy [29]. Using Gauss's law and counterterm methods, we compute the various conserved and thermodynamic quantities of these solutions, obtaining

$$
T=\frac{k}{4 \pi r_{+}}-\frac{r_{+} \Lambda}{4 \pi}-\frac{q^{2}}{4 \pi r_{+}^{3}}+\frac{m^{2}}{4 \pi r_{+}}\left(c c_{1} r_{+}+c_{2} c^{2}\right)
$$




\begin{tabular}{ccc}
\hline \hline$k$ & $m$ & roots \\
\hline \hline 1 & 2.1600 & $3.8955,9.2446$ \\
\hline 0 & 2.1300 & $3.1305,9.3969$ \\
\hline-1 & 2.1600 & $2.6165,10.2691$ \\
\hline \hline 1 & 2.1059 & $0.51778,3.8325,8.4374$ \\
\hline 0 & 2.1291 & $0.5432,3.1271,9.3857$ \\
\hline-1 & 2.1530 & $0.5756,2.5755,10.1809$ \\
\hline \hline 1 & 2.1000 & $0.4381,0.6198,3.8237,8.3482$ \\
\hline 0 & 2.1000 & $0.3753,0.8565,2.9959,9.0022$ \\
\hline-1 & 2.1000 & $0.3449,1.2545,2.1028,9.5275$ \\
\hline \hline
\end{tabular}

Table I: roots of metric function $(\psi(r)=0)$ for $\Lambda=-3, d=4$, $q=1.67, c=1.2, c_{1}=-2, c_{2}=2.2, c_{3}=1.5$ and $m_{0}=34$.

$$
S=\frac{\mathcal{V}_{2} r_{+}^{2}}{4}, \quad Q=\frac{\mathcal{V}_{2} q}{4 \pi}, \quad M=\frac{\mathcal{V}_{2} m_{0}}{8 \pi},
$$

where $\mathcal{V}_{2}$ is the area of a unit volume of constant $(t, r)$ space $(4 \pi$ for $k=0)$. Also the electric potential is

$$
\Phi=\left.A_{\mu} \chi^{\mu}\right|_{r \rightarrow \infty}-\left.A_{\mu} \chi^{\mu}\right|_{r \rightarrow r_{+}}=\frac{q}{r_{+}} .
$$

With these relations, we find that the solutions obey the first law of black hole thermodynamics in an extended phase space (including massive variables)

$$
d M=T d S+\Phi d Q+V d P+\mathcal{C}_{1} d c_{1}+\mathcal{C}_{2} d c_{2}+\mathcal{C}_{3} d c_{3},
$$

where the conjugate quantities associated with the intensive parameters $S, Q, P, c_{i}$ 's are

$$
\begin{aligned}
T & =\left(\frac{\partial M}{\partial S}\right)_{Q, P, c_{i}}, \\
\Phi & =\left(\frac{\partial M}{\partial Q}\right)_{S, P, c_{i}}, \\
V & =\left(\frac{\partial M}{\partial P}\right)_{S, Q, c_{i}}=\frac{\mathcal{V}_{2} r_{+}^{3}}{3}, \\
\mathcal{C}_{1} & =\left(\frac{\partial M}{\partial c_{1}}\right)_{S, Q, P, c_{2}, c_{3}}=\frac{\mathcal{V}_{2} c m^{2} r_{+}^{2}}{16 \pi} \\
\mathcal{C}_{2} & =\left(\frac{\partial M}{\partial c_{2}}\right)_{S, Q, P, c_{1}, c_{3}}=\frac{\mathcal{V}_{2} c^{2} m^{2} r_{+}}{8 \pi} \\
\mathcal{C}_{3} & =\left(\frac{\partial M}{\partial c_{3}}\right)_{S, Q, P, c_{1}, c_{2}}=\frac{\mathcal{V}_{2} c^{3} m^{2}}{8 \pi}
\end{aligned}
$$

with $T$ and $\Phi$ given in Eqs. (8) and (10). In addition, the corresponding Smarr relation can be derived by a scaling (dimensional) argument as

$$
M=2 T S-2 P V+\Phi Q+\mathcal{C}_{1} c_{1}-\mathcal{C}_{3} c_{3},
$$

where $c_{2}$ does not appear since it has scaling weight 0 . Since the $c_{2}$-term in the metric function is a constant term in four dimensions with no thermodynamical contribution, we set $d c_{2}=0$.
We note that the thermodynamic volume (14) does not depend on the graviton mass. This in turn implies that the isoperimetric ratio

$$
\mathcal{R}=\left(\frac{3 V}{\mathcal{V}_{2}}\right)^{\frac{1}{3}}\left(\frac{\mathcal{V}_{2}}{A}\right)^{\frac{1}{2}}=1
$$

and so the reverse isoperimetric inequality $(\mathcal{R} \geq 1)$ 30] can be satisfied.

To study critical phenomena and van der Waals behaviour, we compute the equation of state and the Gibbs free energy

$$
\begin{aligned}
P= & \frac{4 \pi T-m^{2} c_{1} c}{8 \pi r_{+}}-\frac{k+m^{2} c_{2} c^{2}}{8 \pi r_{+}^{2}}+\frac{q^{2}}{8 \pi r_{+}^{4}} \\
G= & H-T S=M-T S=-\frac{P r_{+}^{3}}{6}+ \\
& \frac{m^{2} r_{+}\left(c_{2} c^{2} r_{+}+2 c_{3} c^{3}\right)+\left(k r_{+}^{2}+3 q^{2}\right)}{16 \pi r_{+}}
\end{aligned}
$$

using Eqs. (869). Computing the inflection point $\left(\frac{\partial P}{\partial r_{+}}\right)_{T}=\left(\frac{\partial^{2} P}{\partial r_{+}^{2}}\right)_{T}=0$ of the equation of state, we find

$$
k r_{+c}^{2}-6 q^{2}+m^{2} c_{2} c^{2} r_{+c}^{2}=0 .
$$

where $r_{+c}$ yields the critical volume $V_{c}$ via Eq. (14). This leads to the following respective critical horizon radius, temperature and pressure

$$
\begin{aligned}
r_{+c} & =\frac{\sqrt{6}|q|}{\sqrt{k+m^{2} c_{2} c^{2}}}, \\
T_{c} & =\frac{\left(k+m^{2} c_{2} c^{2}\right)^{3 / 2}}{3 \sqrt{6} \pi q}+\frac{m^{2} c_{1} c}{4 \pi}, \\
P_{c} & =\frac{\left(k+m^{2} c_{2} c^{2}\right)^{2}}{96 \pi q^{2}} .
\end{aligned}
$$

and we see that for all values of $k$ critical behaviour is possible provided the constraint

$$
k+m^{2} c_{2} c^{2} \geq 0
$$

is obeyed. Moreover, taking into account Eq. (20), the pressure is positive for large volume provided

$$
T>\frac{m^{2} c_{1} c}{4 \pi}
$$

a relationship that is automatically satisfied provided (26) holds. Previous investigations of the critical behaviour of black holes in Einstein gravity have indicated that only spherical topology $(k=1)$ admits van der Waals like behaviour, with the $k=0$ case behaving like an ideal gas. The graviton mass significantly modifies this behaviour, opening up new possibilities: topological black holes $(k \neq 1)$ can exhibit second order phase transitions and van der Waals like behaviour (see Fig. 2). This admits new prospects for investigating critical 

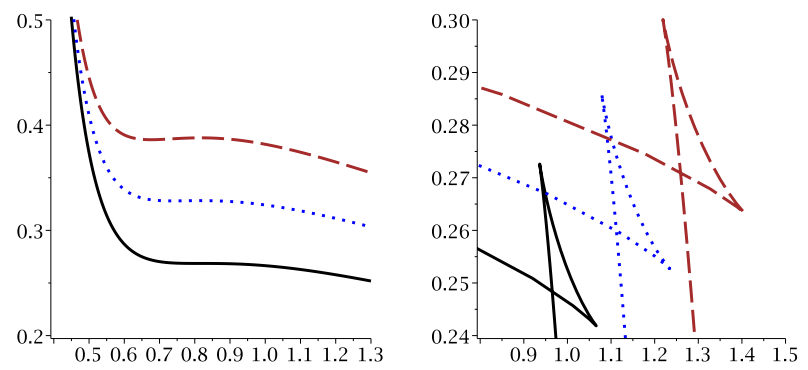

Figure 2: $P-r_{+}$(left panel) and $G-T$ diagrams for $d=4$, $q=m=c=c_{1}=c_{3}=1$ and $c_{2}=10$. Left panel $T=T_{c}$, right panel $P=0.5 P_{c}: k=-1$ (continuous line), $k=0$ (dotted line) and $k=1$ (dashed line).
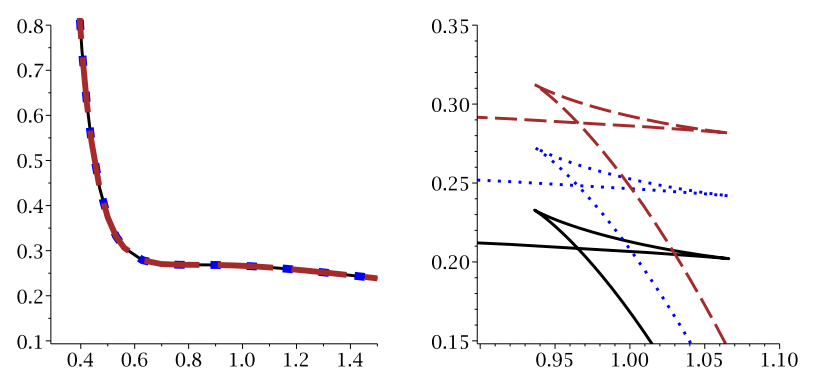

Figure 3: $P-r_{+}$(left panel) and $G-T$ diagrams for $d=4$, $q=m=c=c_{1}=1, k=-1$ and $c_{2}=10$. Left panel $T=T_{c}$, right panel $P=0.5 P_{c}: c_{3}=0$ (continuous line), $c_{3}=1$ (dotted line) and $c_{3}=2$ (dashed line).

behaviour of black holes in the context of classical gravity, the AdS/CFT correspondence, holographic interpretation of black holes, and duality with superconductivity.

The existence of van der Waals like behaviour for nonspherical black holes provides another reason for considering modified versions of Einstein gravity to include massive terms. In other words, a non-zero $m$ admits the possibility of critical behaviour for $k \neq 1$. Furthermore, the massive coefficient $c_{3}$ in the Gibbs free energy (absent in pressure and temperature) makes it possible to modify the energy of different phases, without any modification in critical values and their corresponding diagrams (see Eq. (21) and Fig. 3). For example the formation of a second order phase transition can take place at the same critical temperature, pressure and horizon radius but at differing energies of the various phases. Such interesting behaviors of Gibbs free energy and critical values could introduce new phenomenology for the phase structure of black holes.

Closing Remarks- In this paper we have demonstrated that topological black holes in dRGT can exhibit van der Waals behaviour and critical phenomena, in striking contrast to their counterparts in Einstein gravity. For $k=0$ it is sufficient to have any non-zero value of the graviton mass parameter $m$, whereas for $k=-1$ black holes, this parameter must be sufficiently large. However too large a value of $m$ will destabilize the pressure, causing it to become negative for sufficiently large volume.

Recent progress in gauge/gravity duality in extended phase space [31] suggests that massive gravity will open up avenues of investigation in black hole thermodynamics. Since such theories admit critical behaviour of black holes of any horizon curvature, a range of new phenomena in entanglement entropy [32], holographic ferromagnetism [33], quasinormal modes [34], and confinement/deconfinment phase transitions for heavy quarks [35] can now be explored.

We gratefully thank the anonymous referees for enlightening comments and suggestions which substantially helped in improving the quality of the paper. SHH and BE thank Shiraz University Research Council. The work of RBM was supported by the Natural Science and Engineering Research Council of Canada. This work has been supported financially by the Research Institute for Astronomy and Astrophysics of Maragha, Iran.
[1] M. Fierz and W. Pauli, Proc. Roy. Soc. Lond. A 173, 211 (1939).

[2] D. G. Boulware and S. Deser, Phys. Rev. D 6, 3368 (1972).

[3] C. de Rham and G. Gabadadze, Phys. Rev. D 82, 044020 (2010); C. de Rham, G. Gabadadze and A. J. Tolley, Phys. Rev. Lett. 106, 231101 (2011); S. F. Hassan and R. A. Rosen, JHEP 07, 009 (2011).

[4] S. F. Hassan and R. A. Rosen, Phys. Rev. Lett. 108, 041101 (2012); S. F. Hassan, R. A. Rosen and A. Schmidt-May, JHEP 02, 026 (2012).

[5] M. Fasiello and A. J. Tolley, JCAP 12, 002 (2013); E. Babichev and A. Fabbri, Phys. Rev. D 90, 084019 (2014); K. Bamba, Md. W. Hossain, R. Myrzakulov, S. Nojiri and M. Sami, Phys. Rev. D 89, 083518 (2014); G. Goon, A. E. Gumrukcuoglu, K. Hinterbichler, S. Mukohyama and M. Trodden, JCAP 08, 008 (2014); L. Heisenberg, R. Kimura and K. Yamamoto, Phys. Rev. D 89, 103008 (2014); A. R. Solomon, J. Enander, Y. Akrami, T. S. Koivisto, F. Konnig and E. Mortsell, JCAP 04, 027 (2015); P. Li, X. Zh. Li and P. Xi, Phys. Rev. D 93, 064040 (2016); S. G. Ghosh, L. Tannukij and P. Wongjun, Eur. Phys. J. C 76, 119 (2016).

[6] C. de Rham, Living Rev. Rel. 17, 7 (2014).

[7] D. Vegh, arXiv:1301.0537.

[8] H. Zhang and X. Z. Li, Phys. Rev. D 93, 124039 (2016).

[9] S. H. Hendi, B. Eslam Panah and S. Panahiyan, JHEP 11, 157 (2015); J. Xu, L. M. Cao and Y. P. Hu, Phys. Rev. D 91, 124033 (2015); S. H. Hendi, B. Eslam Panah and S. Panahiyan, JHEP 05, 029 (2016).

[10] S. H. Hendi, S. Panahiyan and B. Eslam Panah, JHEP 01, 129 (2016). 
[11] D. Hansen, N. Yunes and K. Yagi, Phys. Rev. D 91, 082003 (2015).

[12] A. Gruzinov, New Astronomy, 10, 311 (2005); A. S. Goldhaber and M. M. Nieto, Rev. Mod. Phys. 82, 939 (2010); C. de Rham, J. T. Deskins, A. J. Tolley and S. Y. Zhou, arXiv:1606.08462

[13] LIGO Scientific Collaboration, Phys. Rev. Lett. 116, 061102 (2016); LIGO Scientific Collaboration, Phys. Rev. Lett. 116, 221101 (2016).

[14] L. S. Finn and P. J. Sutton, Phys. Rev. D 65044022 (2002).

[15] J. B. Jimenez, F. Piazza and H. Velten, Phys. Rev. Lett. 116, 061101 (2016).

[16] S. W. Hawking and D. N. Page, Commun. Math. Phys. 87, 577 (1983).

[17] E. Witten, Adv. Theor. Math. Phys. 2, 253 (1998); O. Aharony, S. S. Gubser, J. M. Maldacena, H. Ooguri and Y. Oz, Phys. Rept. 323, 183 (2000).

[18] S. A. Hartnoll, Class. Quant. Grav. 26, 224002 (2009); S. S. Lee, arXiv:1009.5127, J. McGreevy, Adv. High Energy Phys. 2010, 723105 (2010); S. Sachdev, Lecture Notes in Physics, 828, 273 (2011).

[19] B. P. Dolan, Class. Quant. Grav. 28, 125020 (2011); D. Kubiznak and R. B. Mann, JHEP 07, 033 (2012).

[20] N. Altamirano, D. Kubiznak, R. B. Mann and Z. Sherkatghanad, Class. Quant. Grav. 31, 042001 (2014); S. W. Wei and Y. X. Liu, Phys. Rev. D 90, 044057 (2014); R. A. Hennigar, W. G. Brenna and R. B. Mann, JHEP 07, 077 (2015).

[21] S. Gunasekaran, R. B. Mann and D. Kubiznak, JHEP 11, 110 (2012); A. M. Frassino, D. Kubiznak, R. B. Mann and F. Simovic, JHEP 09, 080 (2014); R. A. Hennigar and R. B. Mann, Entropy 17, 8056 (2015).

[22] C. V. Johnson, Class. Quant. Grav. 31, 205002 (2014);

A. Belhaj, M. Chabab, H. El Moumni, K. Masmar, M. B. Sedra and A. Segui, JHEP 05, 149 (2015).

[23] Z. Y. Nie and H. Zeng, JHEP 10, 047 (2015).

[24] M. Guica, T. Hartman, W. Song and A. Strominger, Phys. Rev. D 80, 124008 (2009); M. Sinamuli and R. B. Mann, JHEP 08, 148 (2016).

[25] D. Kastor, S. Ray and J. Traschen, JHEP 11, 120 (2014); D. Kastor, S. Ray and J. Traschen, Entropy 18, 212 (2016).

[26] D. Kubiznak, R. B. Mann and Mae Teo, arXiv:1608.06147

[27] R. G. Cai, Y. P. Hu, Q. Y. Pan and Y. L. Zhang, Phys. Rev. D 91, 024032 (2015).

[28] S. Nojiri and S. D. Odintsov, Int. J. Mod. Phys. A 14, 1293 (1999); R. Casadio and C. Germani, Prog. Theor. Phys. 114, 23 (2005).

[29] D. Kastor, S. Ray and J. Traschen, Class. Quant. Grav. 26, 195011 (2009).

[30] M. Cvetic, G. W. Gibbons, D. Kubiznak and C. N. Pope, Phys. Rev. D 84, 024037 (2011).

[31] A. Karch and B. Robinson, JHEP 12, 073 (2015); X. X. Zeng and L. F. Li, arXiv:1512.08855

[32] E. Caceres, P. H. Nguyen and J. F. Pedraza, JHEP 09, 184 (2015); S. Kundu and J. F. Pedraza, arXiv:1602.07353

[33] R. G. Cai, R. Q. Yang, Y. B. Wu and C. Y. Zhang, JHEP 11, 021 (2015).

[34] Y. Liu, D. C. Zou and B. Wang, JHEP 09, 179 (2014).

[35] Y. Yang and P. H. Yuan, JHEP 12, 161 (2015). 